\title{
Liquid-liquid transition in supercooled silicon determined by first-principles simulation
}

\author{
P. Ganesh \\ Carnegie Institution of Washington, Washington DC \\ M. Widom \\ Department of Physics, Carnegie Mellon University, Pittsburgh, PA
}

(Dated: October 26, 2018)

\begin{abstract}
First principles molecular dynamics simulations reveal a liquid-liquid phase transition in supercooled elemental silicon. Two phases coexist below $T_{c} \approx 1232 \mathrm{~K}$. The low density phase is nearly tetra-coordinated, with a pseudogap at the Fermi surface, while the high density phase is more highly coordinated and metallic in nature. The transition is observed through the formation of van der Waals loops in pressure-volume isotherms below $T_{c}$.

PACS numbers:
\end{abstract}

Silicon occupies a position in the periodic table at the border between metals and insulators. At low pressure, crystalline silicon (c-Si) is tetrahedrally coordinated (like diamond) and is an indirect band-gap semiconductor. As pressure increases, crystalline Si transforms from the diamond phase through a more highly-coordinated metallic $\beta$-tin phase to a hexagonal phase. Like c-Si, noncrystalline amorphous silicon (a-Si) is semiconducting at low pressure and maintains a coordination number $N_{c} \approx 4$. At higher pressures, amorphous silicon becomes metallic and more highly coordinated. Thus, both c- and a-Si exhibit pressure-induced polymorphism.

Liquid silicon (l-Si) is metallic at high temperature, although it possesses a small population of covalent bonds [1, 2]. The coordination number $N_{c} \approx 7.3$ (for liquid and amorphous structures $N_{c}$ is the average number of neighbors within the first peak of the radial distribution function $g(r))$ is lower than occurs in ordinary metals such as aluminum or copper, but higher than in c-Si. As expected from its higher coordination number, l-Si has higher density than c-Si, a property that silicon shares with water [3]. 
The structure of supercooled l-Si and its transition from a metallic dense-packed structure at high temperature to a semiconducting open network at low temperature remains uncertain. The dissimilarity of the amorphous and liquid states raises the possibility of intermediate phases [4]. By analogy with the liquid-liquid phase transition (LLPT) in water [5, 6], where tetrahedral order is also present in the crystalline solid phase, researchers speculate [7] such a transition might occur in silicon, between a tetracoordinated low density liquid (LDL) and a more highly coordinated high density liquid (HDL). Since the LLPT presumably occurs in the supercooled liquid, both LDL and HDL are metastable states, if they exist at all. The supercooled HDL state is the metastable extension of the high temperature equilibrium liquid (HTEL) state, while the glassy a-Si state is the extension of LDL to low temperature.

Experimental evidence for an LLPT is not conclusive. Pressure induced transformation of a-Si [8], from a low-density amorphous (LDA) state at low pressure to a high-density amorphous state (HDA), similar to that of amorphous ice [9], suggests the possibility of an LLPT in silicon similar to that in supercooled water. Plastic deformation [10] studies indicate a transition from a-Si to a fluid state, tentatively identified as LDL, at a temperature around $1000 \mathrm{~K}$ (the melting point is $T_{m}=1687 \mathrm{~K}$ ). Containerless supercooling experiments [11, $12,13,14,15]$ lead to contradictory results regarding the temperature dependence of the supercooled liquid properties.

Computer simulations reveal complete structural detail not available by ordinary experimental means. However, they suffer from uncertainty arising from approximate models of interatomic forces and energetics, as well as limitations of sample size and simulation time. To capture the nature of tetrahedral bonding, the Stillinger-Weber (S-W) potential [16] includes angle dependent interactions. This potential shows clear evidence of a first-order HDL to LDL transition, in the deeply supercooled regime (with a transition temperature $\left.T_{c} \sim 1060 \mathrm{~K}\right)$ [17]. Electronic structure calculations on these simulated configurations report a metal to semimetal transition across the LLPT transition temperature [18]. Other empirical potentials, such as the Keating potential [19] and the environment dependent potential [20], find contradictory results, so the nature and existence of an LLPT depends on the form of the interaction model [21].

Since neither experiments nor simulations with empirical potentials resolve the existence or character of an LLPT in Si with certainty, further investigation using first-principles 
methods is warranted. The pioneering Car-Parrinello simulations [1, 22] studied solid c$\mathrm{Si}$ and a-Si in addition to high temperature l-Si. A later simulation of the supercooled liquid [23] in a small $N=64$ atom cell found increased tetrahedral order and a sudden drop in $N_{c}$ at $\mathrm{T}=1100 \mathrm{~K}$. Our study carries out a more extensive investigation and finds van der Waals loops in the pressure-volume isotherms (see Fig. 1), a strong indication of an LLPT, with a critical point around $T_{c} \approx 1232 \mathrm{~K}$.

Our simulations use the Vienna Ab-Initio Simulation Package (VASP) [24, 25] together with Projector Augmented Wave (PAW) potentials [26, 27] in the Generalized Gradient Approximation (GGA). Electronic structure calculations of energies, pressures and forces used the $\Gamma k$-point only and were conducted at the default energy cutoff of $245 \mathrm{eV}$. Molecular dynamics utilized a $2 \mathrm{fs}$ time step, and velocities were rescaled every time step to maintain constant temperature. Our molecular dynamics calculations extended to 3ps or more and required 2 weeks of computer time for each temperature and volume.

To test for finite size effects we evaluated the radial distribution function $g(r)$ at system sizes $N=100,200$ and 300 and found adequate convergence at $N=200$ atoms. We transformed $g(r)$ to obtain the structure factor $S(q)$, using the Baxter method [28] (see Fig. 22). The peak and shoulder positions agree well with experiment [29], but the experimental $S(q)$ exhibits a slight excess at low $q$ arising from a background effect, causing the experimental data to violate a sum rule associated with short distance correlations [28], while the simulation data obey the sum rule.

Ideally a phase transition should be observed as a function of temperature $T$ and pressure $P$. However, the VASP program is restricted to fixed volume simulation, so we monitored the reported "external pressure" (derivative of potential energy with respect to volume [30]) and added the kinetic energy contribution $\rho k_{B} T$ to obtain the thermodynamic pressure. We also add a constant offset of $P_{\text {Pulay }}=1.4$ kilobar to account for the Pulay stress, which we found to be reasonably independent of temperature and volume.

At least 9 temperatures were studied at each volume, uniformly spaced between a low of $\mathrm{T}=982 \mathrm{~K}$ and a high of $1382 \mathrm{~K}$, with additional intermediate temperatures included in cases where the mean internal energy varied rapidly with temperature. Each starting configuration for a given $\mathrm{V}$ and $\mathrm{T}$ was generated by a lengthy $\mathrm{MD}$ simulation prior to the beginning of data collection. To improve the efficiency of configuration sampling, we employed a replica exchange method [28, 31, 32] to swap configurations between temperatures. Briefly, 
pairs of configurations at common volume but differing in energy by $\Delta E$ and differing in inverse temperature $\left(\beta=1 / k_{B} T\right)$ by $\Delta \beta$, were swapped with probability $\exp (\Delta \beta \Delta E)$. We attempted swaps every $100 \mathrm{fs}$.

Having energy and pressure data at a series of nearby temperatures allows us to employ the "multiple histogram" technique [32, 33]. Histograms of internal energy at fixed temperature $H_{T}(E)$ are converted into configurational densities of states $\Omega(E)=$ $\left(\sum_{T} H_{T}(E)\right) /\left(\sum_{T} e^{(F(T)-E) / k_{B} T}\right)$ where the sums run over the discrete temperatures $T$ at which simulations were performed. Consistency between temperatures is enforced by their free energies $F(T)=-k_{B} T \ln Z(T)$ where the partition function $Z(T)=\int \Omega(E) e^{-E / k_{B} T} \mathrm{~d} E$. We thus reconstruct the free energy and its derivative quantities such as pressure and heat capacity as analytic functions of temperature. For example the pressure is obtained from

$$
P(T)=\frac{1}{Z(T)} \int \bar{P}(E) \Omega(E) e^{-E / k_{B} T} \mathrm{~d} E
$$

where $\bar{P}(E)$ is the mean pressure observed at energy $E$. Accuracy of this method depends on a thorough sampling of configuration space and on sufficient overlap of the energy histograms at adjacent temperatures.

Results for the pressure are illustrated in Fig. 1. Error bars are estimates of standard error, defined as the RMS fluctuation in pressure divided by $\sqrt{N_{\text {ind }}}$, where we set $N_{\text {ind }}=10$ as the approximate number of independent samples. Two main features of this plot are: van der Waals loops, and negative thermal expansion.

Van der Waals loops occur when a region of positive slope interrupts the generally negative slope of the pressure-volume isotherm. Positive slope of $P(V)$ corresponds to negative isothermal compressibility $\left(K_{T}=-\frac{1}{V}\left(\frac{\partial V}{\partial P}\right)_{T}\right)$. This thermodynamically unstable state would phase separate into high volume (low density) and low volume (high density) phases if the system size were sufficiently large. However, negative $K_{T}$ is permissible in systems of finite size owing to the free energy cost of the interface needed to separate the two phases. Maxwell's equal area construction can be used to identify the coexisting states below the critical temperature, which we identify as $T_{c} \approx 1232 \mathrm{~K}$. At $T=1182 \mathrm{~K}$, we find coexisting states: HDL at density $\rho=0.053$ atoms $/ \AA^{3}$ (volume $v=18.9 \AA^{3} /$ atom) with coordination number $N_{c}=5.6$; LDL at density $\rho=0.049(v=20.4)$ with coordination number $N_{c}=4.2$. According to the isotherms, HDL is the extension of the equilibrium liquid.

Negative thermal expansion $\left(\alpha_{P}=\frac{1}{V}\left(\frac{\partial V}{\partial T}\right)_{P}\right)$ occurs when the pressure is a decreasing 
function of temperature and can be seen at volumes above $18 \AA^{3} /$ atom. Negative thermal expansion is thermodynamically permissible, and is seen in water close to freezing, for example, but is quite rare. Thermal expansion is related to entropy via $\left(\frac{\partial S}{\partial V}\right)_{T}=\frac{\alpha_{P}}{K_{T}}$. Since LDL exhibits negative $\alpha_{P}$, we see that entropy decreases (counterintuitively) as volume increases. Presumably this reflects the higher degree of orientational order in the open tetracoordinated network as compared with the disordered high density liquid state [34].

Fig. 2 (inset) shows the distribution of the tetrahedral order parameter [3],

$$
q_{t}=1-\frac{3}{8} \sum_{i<j=1}^{4}\left(\cos \theta_{i j}+\frac{1}{3}\right)^{2}
$$

where $\theta_{i j}$ is the angle formed by an atom with its $i^{\text {th }}$ and $j^{\text {th }}$ nearest neighbors. A value close to 1 indicates tetrahedral bonding. We evaluate this distribution in the HDL and LDL states at temperature $\mathrm{T}=1182 \mathrm{~K}$. At lower densities, the favored LDL structure is indeed much more tetrahedral than the higher density HDL or HTEL structures.

Because the LLPT occurs between metastable liquid phases, below the equilibrium freezing transition, a crucial question is if our coexisting phases at low temperature are both truly liquid. Indeed, since LDL exhibits fairly strong peaks in $g(r)$ and $S(q)$ as well as a high degree of tetrahedral order, a central concern is if this LDL phase has not actually crystallized. Likely candidates for the crystal structure are the usual c-Si diamond structure of Pearson type $\mathrm{cF} 8$, or the Pearson type cI16 crystal that arises upon recovery at atmospheric pressure following high pressure treatment.

To make direct comparisons we carried out solid-state molecular dynamics simulations. For cF8 we took $N=216$ atoms at $v=20.4 \AA^{3}$, and for cI16 we took $N=192$ atoms at $v=18.5 \AA^{3}$, the densities being chosen taking thermal expansion into account. Comparisons of $g(r)$ and $S(q)$ are shown in Fig. 3. In order to smear out the Bragg peaks in $S(q)$ we smoothly truncated the crystalline $g(r)$ prior to Fourier transformation, so the figures understate the strength of peaks in $S(q)$ for the crystalline structures. At $T=1182 \mathrm{~K}$ both crystal structures remain far better ordered than the LDL. Additionally, for both $g(r)$ and $S(q)$ there is no systematic resemblance between LDL and either crystal structure.

Comparing $q_{t}$ distributions at $\mathrm{T}=1182 \mathrm{~K}$ (not shown) we found the distribution for $\mathrm{cF} 8$ was more sharply peaked close to $q_{t}=1$ than that for LDL, but the distribution for cI16 was rather similar to that of LDL. Coordination numbers also were similar between LDL and cI16. We examined the statistics of atom rings. For the ideal crystal structures, both 
cF8 and cI16 have only even length rings, and no rings of length less than 6. For purposes of comparison we take the ratio of the number of rings with length 5 or 7 to the number with length 6 or 8 . Values of $\left(N_{5}+N_{7}\right) /\left(N_{6}+N_{8}\right)$ at $T=1182 \mathrm{~K}$ were: 0.00 for $\mathrm{cF} 8,0.08$ for cI16 and 0.50 for LDL. We also found some rings of length 3 and 4 in LDL, but not in either cF8 or cI16. Finally, we visually inspected atomic configurations and saw no hints of crystallinity in LDL

The electronic density of states (DOS) governs important material properties including electric conductivity. Fig. 4 illustrates the DOS of the equilibrium HTEL, metastable LDL and HDL, and c-Si states. Liquid state DOS are presented as the average of five independent configurations. The Fermi energies $E_{F}$ of HTEL and HDL are similar because their atomic volumes are similar. Likewise the $E_{F}$ 's are similar for LDL and $\mathrm{cF} 8$, but both lie below those of HTEL and HDL because their atomic volumes are greater than for HDL and HTEL. The bottom of the LDL valence band (around $-7 \mathrm{eV}$ ) lies slightly below $\mathrm{cF} 8$, reflecting the slightly higher coordination of LDL. HTEL and HDL have high densities of states at $E_{F}$ implying metallic character. LDL has a deep pseudogap at $E_{F}$ suggesting semimetallic character [18]. c-Si exhibits the characteristic bandgap of a semiconductor.

In conclusion, we find that deeply supercooled liquid silicon undergoes a liquid-liquid phase transition, separating into a high density highly coordinated metallic liquid and a low density low coordinated semimetallic liquid. Our calculation reveals structural detail not currently available from experiment in this temperature range. Because we employ first-principles forces, we have confidence in the validity of the model. The critical point lies close to $T=1232 \mathrm{~K}$, not far from estimates from experiment [8, 10] and simulations based on empirical potentials [17]. HTEL and HDL share characteristics such as low atomic volume, high coordination, moderate $q_{t}$ and metallic conduction, consistent with the notion that HDL is the metastable extension of HTEL into the supercooled regime. Likewise c-Si and LDL share characteristics such as high atomic volume, low coordination, large $q_{t}$ and low DOS at $E_{F}$, consistent with LDL exhibiting local tetrahedral order similar to c-Si. 


\section{Acknowledgments}

We wish to thank Bob Swendsen for numerous helpful discussions. We also thank Ken Kelton and Alan Goldman for sharing the experimental data plotted in Fig. 2,

[1] I. Stich, R. Car, and M. Parrinello, Phys. Rev. Lett. 63, 2240 (1989).

[2] W. Jank and J. Hafner, Phys. Rev. B 41, 1497 (1990).

[3] J. R. Errington and P. G. Debenedetti, Nature 409, 318 (2001).

[4] L. I. Aptekar, Sov. Phys. Dokl. 24, 993 (1979).

[5] P. H. Poole, F. Sciortino, U. Essmann, and H. E. Stanley, Nature 360, 324 (1992).

[6] O. Mishima and H. E. Stanley, Nature (London) 396, 329 (1998).

[7] P. H. Poole, T. Grande, C. A. Angell, and P. F. McMillan, Science 275, 322 (1997).

[8] S. K. Deb, M. Wilding, M. Somayazulu, and P. F. McMillan, Nature 414, 528 (2001).

[9] O. Mishima, L. D. Calvert, and E. Whalley, Nature 314, 76 (1985).

[10] A. Hedler, S. L. Klaumunzer, and W. Wesch, Nature Mat. 3, 804 (2004).

[11] S. Ansell, S. Krishnan, J. J. Felten, and D. L. Price, J. Phys.: Cond. Matter 10, L73 (1998).

[12] N. Jakse, N. Hennet, D. L. Price, S. Krishnan, T. Key, E. Artacho, B. Glorieux, A. Pasturel, and M. L. Saboungi, App. Phys. Lett. 83, 4734 (2003).

[13] H. Kimura, M. Watanabe, and K. Izumi, App. Phys. Lett. 78, 604 (2001).

[14] K. Higuchi, K. Kimura, A. Mizuno, M. Watanabe, Y. Katayama, and K. Kuribayashi, Meas. Sci. Tech. 16, 381 (2005).

[15] T. H. Kim, G. W. Lee, B. Sieve, A. K. Gangopadhyay, R. W. Hyers, T. J. Rathz, J. R. Rogers, D. S. Robinson, K. F. Kelton, and A. I. Goldman, Phys. Rev. Lett. 95, 085501 (2005).

[16] F. H. Stillinger and T. A. Weber, Phys. Rev. B 31, 5262 (1985).

[17] S. Sastry and C. A. Angell, Nature Material 2, 739 (2003).

[18] S. S. Ashwin, U. V. Waghmare, and S. Sastry, Phys. Rev. Lett. 92, 175701 (2004).

[19] Y. Tu, J. Tersoff, G. Grinstein, and D. Vanderbilt, Phys. Rev. Lett. 81, 4899 (1998).

[20] C. R. Miranda and A. Antonelli, J. Chem. Phys. 120, 11672 (2004).

[21] P. Beaucage and N. Mousseau, J. Phys.: Cond. Matter 17, 2269 (2005).

[22] R. Car and M. Parrinello, Phys. Rev. Lett. 55, 2471 (1985). 
[23] T. Morishita, Phys. Rev. Lett. 97, 165502 (2006).

[24] G. Kresse and J. Hafner, Phys. Rev. B 47, R558 (1993).

[25] G. Kresse and J. Furthmuller, Phys. Rev. B 54, 11169 (1996).

[26] P. E. Blochl, Phys. Rev. B 50, 17953 (1994).

[27] G. Kresse and D. Joubert, Phys. Rev. B 59, 1758 (1999).

[28] P. Ganesh and M. Widom, Phys. Rev. B 77, 014205 (2008).

[29] K. F. Kelton, G. W. Lee, A. K. Gangopadhyay, R. W. Hyers, T. J. Rathz, J. R. Rogers, M. B. Robinson, and D. S. Robinson, Phys. Rev. Lett. 90, 195504 (2003).

[30] O. H. Nielsen and R. M. Martin, Phys. Rev. B 32, 3780 (1985).

[31] R. H. Swendsen and J. S. Wang, Phys. Rev. Lett. 57, 2607 (1986).

[32] M. E. J. Newman and G. T. Barkema, Monte Carlo Methods in Statistical Physics (Oxford, 1999).

[33] A. M. Ferrenberg and R. H. Swendsen, Phys. Rev. Lett. 63, 1195 (1989).

[34] P. G. Debenedetti and H. E. Stanley, Physics Today 56, 40 (2003). 


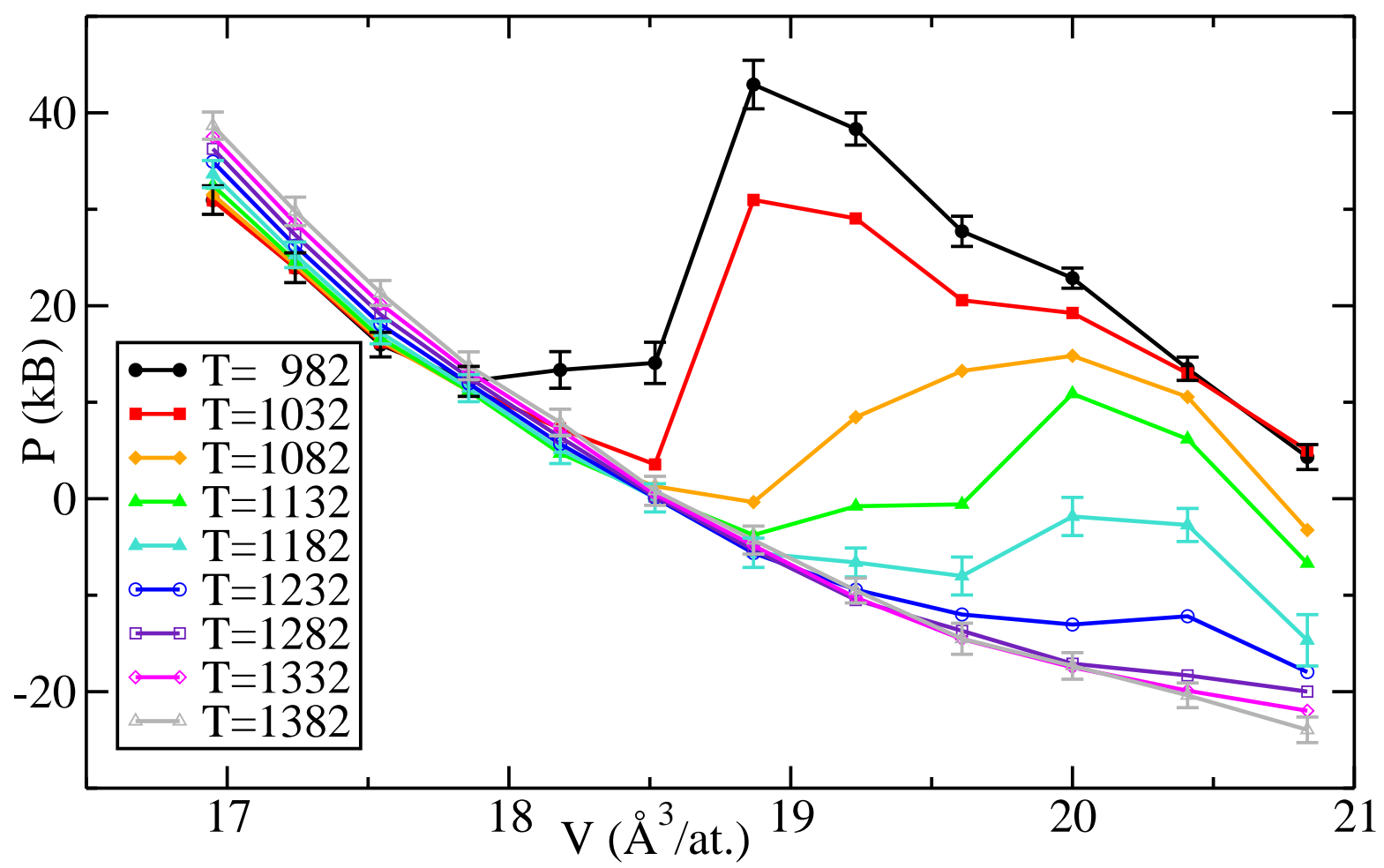

FIG. 1: (color online) Pressure-volume isotherms of liquid Si. Data points are calculated using Eq. 1. 


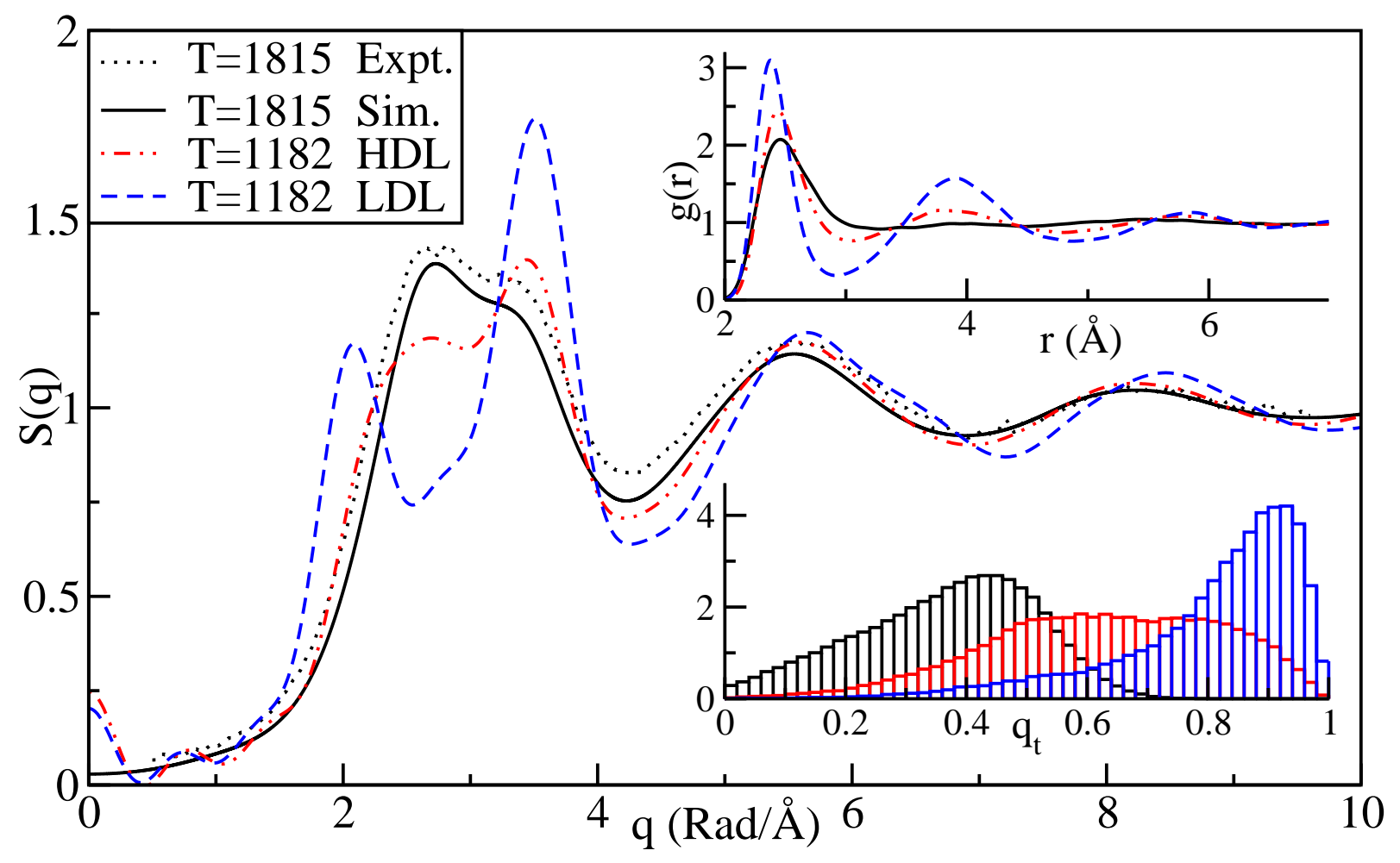

FIG. 2: (color online) Liquid structure factors $S(q)$, radial distribution functions $g(r)$ and tetrahedral order parameter $\left(q_{t}\right)$ distributions. High temperature simulation at temperature $T=1815 \mathrm{~K}$ and volume per atom $v=18.2 \AA^{3}$ is compared with experiment [29]. Low temperature simulation at $T=1182 \mathrm{~K}$ shows coexisting structures, HDL at $v=18.9 \AA^{3}$ and LDL at $v=20.4 \AA^{3}$. 


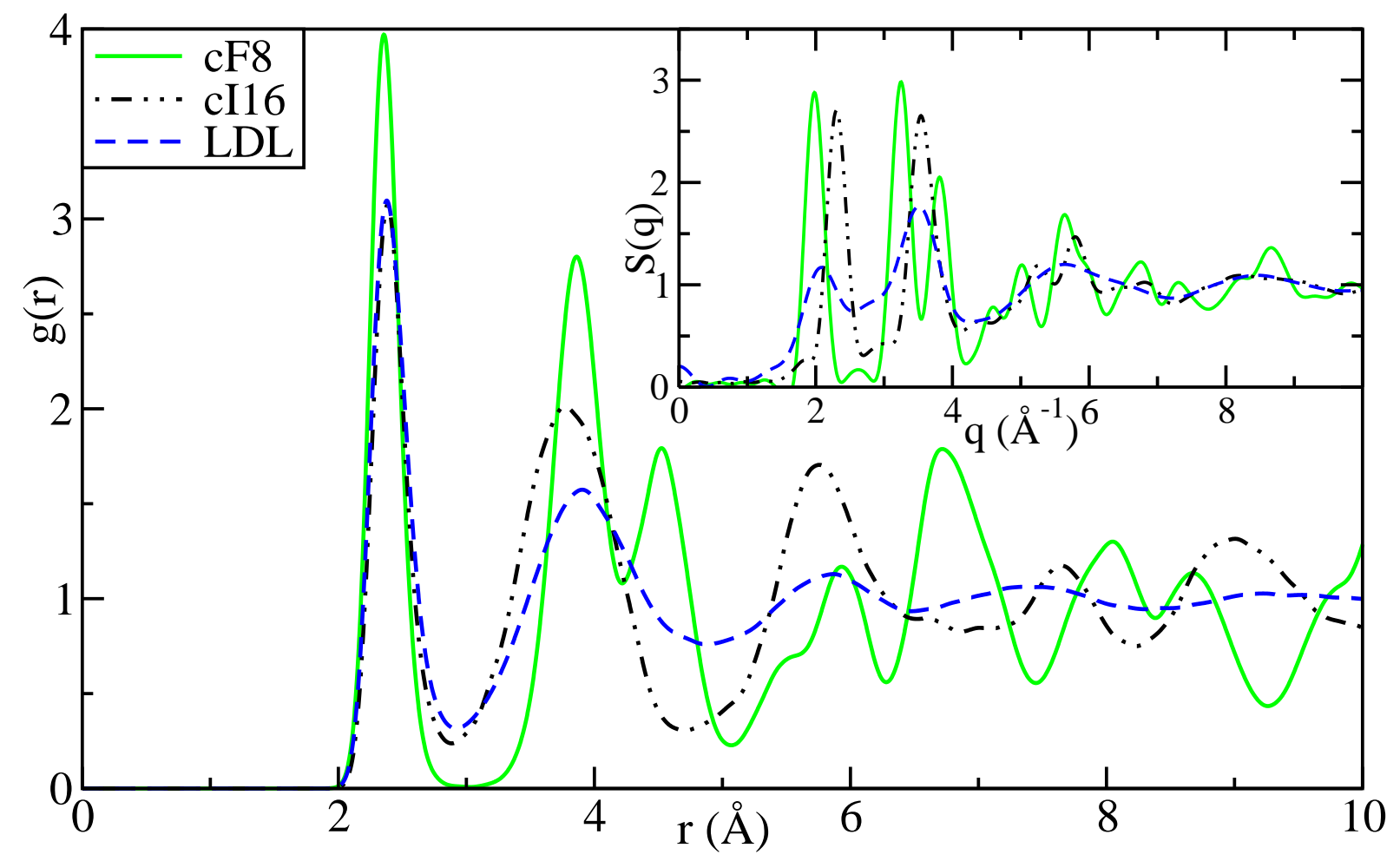

FIG. 3: (color online) Comparison of low-density liquid structure with competing crystal structures at $T=1182 \mathrm{~K}$. 


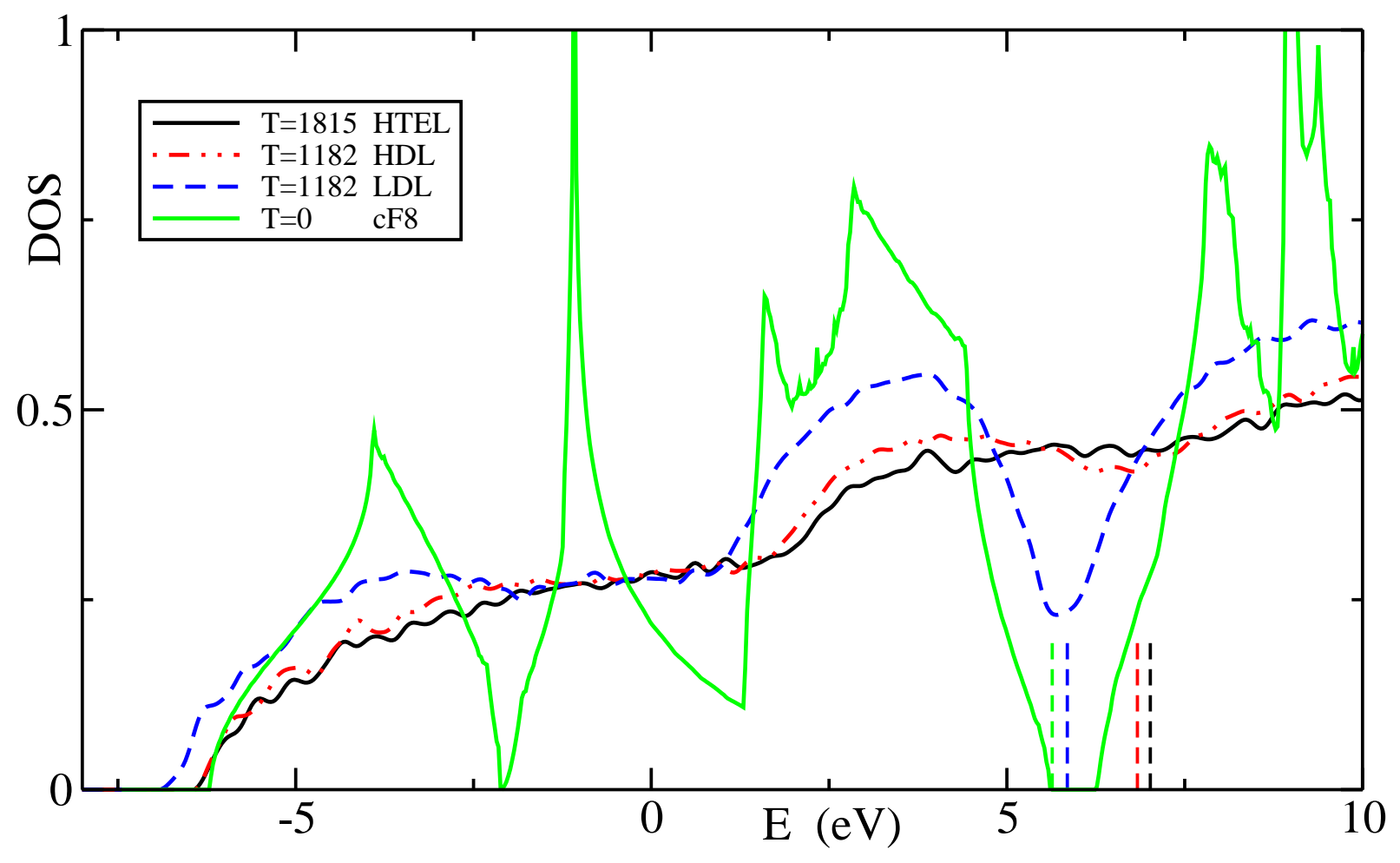

FIG. 4: (color online) Electronic densities of states (states/eV/atom). Vertical dashed lines locate $E_{F}$. 